\title{
Activation of glucagon-like peptide-1 receptors might be a mechanism of the beneficial effects of oral galactose in streptozotocin-induced rat model of sporadic Alzheimer's disease
}

\author{
Jelena Osmanovic Barilar ${ }^{1}$, Ana Knezovic ${ }^{1}$, Ana Babic ${ }^{1}$, Peter Riederer ${ }^{2,3}$, Melita Salkovic-Petrisic ${ }^{1}$ \\ ${ }^{I}$ Department of Pharmacology, University of Zagreb School of Medicine, Zagreb, Croatia, ${ }^{2}$ Center of Mental Health, \\ Department of Psychiatry, Psychosomatics and Psychotherapy, University Hospital, Wuerzburg, Germany, ${ }^{3}$ Department \\ and Research Unit of Psychiatry, Institute of Clinical Research, University of Southern Denmark, Odense, Denmark
}

\section{Background}

Insulin resistance in the brain (IRB) and cerebral glucose hypometabolism are considered to be the pathophysiological core of sporadic Alzheimer's disease ( $\mathrm{sAD}$ ) and drugs aiming to improve these metabolic alterations are currently being investigated. Galactose is intracellularly converted to glucose and may act like an alternative source of energy compensating for dysfunction of insulin-dependent glucose transporters (GLUT) in IRB state. Orally given galactose prevented development of cognitive deficits in streptozotocin-induced AD rat model (STZ-icv) in our previous research. This study aimed to investigate whether galactose induced cognitive improvement in STZ-icv rat model has been mediated by changes in glucagon-like peptide 1 receptor (GLP-1R), and glucose transporters GLUT3 and GLUT4 in the brain.

\section{Methods}

Adult male Wistar rats were given STZ (3 mg/kg) or vehicle (controls) icv bilaterally into lateral ventricles. In the acute experiment single galactose dose was given intraperitoneally $(10$ and $200 \mathrm{mg} / \mathrm{kg}$ ) or orally $(200$ and $1000 \mathrm{mg} / \mathrm{kg})$ to control and STZ rats one month after icv-injection and animals were sacrificed 15 min after galactose administration. In the chronic experiment 2-month oral galactose treatment $(200 \mathrm{mg} / \mathrm{kg})$ was initiated 1 month after STZ-icv. Hippocampal (HPC) expression of GLP-1R, GLUT3 and GLUT4 was analyzed by Western blot and hypothalamic (HPT) expression of GLP-1R was assessed by immunohistochemistry. Data were analyzed by Kruskall-Wallis median test followed by ManneWhitney U-test $(\mathrm{p}<0.05)$.

Results

Acute oral or intraperitoneal galactose load increased GLUT3 expression (+90\%) in HPC of STZ-icv rats but had no effects on GLP-1R expression while chronic oral galactose treatment increased both GLP-1R (+40\%) and GLUT3 $(+60 \%)$ expression in HPC of STZ-icv rats. GLUT4 expression remained unchanged in acute and chronic galactose treatment. GLP-1R expression in HPT was increased in STZ-icv rats both after acute and chronic galactose treatment.

Conclusions

Our findings indicate beneficial effects on GLUT3 and give evidence on GLP-1-mediated effects of oral galactose that might provide a mechanism of the neuroprotective activity of chronic oral galactose on cognitive impairment observed in our research in STZ-rat model of sAD. These findings might open a new, nutrient-based strategy in sAD treatment.

Supported by HRZZ project; IP-2014-09-4639 